\title{
TRASTORNOS DEL APRENDIZAJE: INVESTIGACIONES PSICOLÓGICAS Y PSICOPEDAGÓGICAS EN DIVERSOS PAÍSES DE SUD AMÉRICA*
}

\section{LEARNING DISORDERS: PSYCHOLOGICAL AND PSYCHOPEDAGOGICAL RESEARCH IN DIFFERENT SOUTH AMERICAN COUNTRIES}

\author{
Luis Bravo-Valdivieso \\ Neva Milicic-Müller \\ Universidad Católica de Chile, Chile \\ Ariel Cuadro \\ Universidad Católica del Uruguay, Uruguay \\ Lydia Mejía \\ Instituto Colombiano de Neurociencias, Colombia \\ Jorge Eslava \\ Presidente de la Sociedad Latinoamericana de Neuropsicología

\begin{abstract}
Resumen: El presente artículo hace una reseña del origen de las investigaciones y estrategias de trabajo con niños que presentan Dificultades de Aprendizaje en varios países de Sud América. Aborda inicialmente las investigaciones clínicas neurológicas y psicológicas y luego los estudios y estrategias psicopedagógicas para el trabajo en las escuelas. Su objetivo es mostrar una visión histórica de las distintas estrategias que ha elaborado cada uno de los países estudiados.
\end{abstract}

Palabras clave: dificultades de aprendizaje, revisión, intervención psicopedagógica.

\begin{abstract}
The present study addresses a review on the origin of research and work strategies implemented on children with Learning Difficulties in several South American countries. Initially, it describes the clinical neurological and psychological investigations, outlining afterwards researches and psychopedagogic strategies conducted at schools. The aim is to bring to light a historical perspective of the different strategies that have been developed in each of the studied countries.
\end{abstract}

Keywords: learning difficulties, review, psychopedagogical intervention

* Trabajo de Revisión (N.E.).

\section{ANTECEDENTES HISTÓRICOS}

El conocimiento de los orígenes de una ciencia o de las investigaciones que la configuran es el punto de partida para la comprensión de su nivel de conocimiento actual y de la validez de los estudios efectuados a lo largo de los años.

Las investigaciones científicas no emergen en forma espontánea, sino que son resultantes de una maduración personal de algunos investigadores y del intercambio de informaciones entre especialistas, sobre metodologías y modelos teóricos que las sustentan. Estos intercambios adquieren mayor validez cuando se efectúan en el campo internacional.
El objetivo de este artículo es describir algunas investigaciones que han tenido mayor relevancia en algunos países sudamericanos y cuyos resultados pueden aportar información intercambiable entre los investigadores de los diferentes países involucrados. En ningún caso pretende ser una descripción exhaustiva de ellos, sino solamente mostrar que en los países considerados ha habido investigaciones históricas que han contribuido al progreso de la psicología y de la educación. Su análisis está limitado por la carencia y pobreza de información sobre lo que ha sucedido en diversos países, especialmente en los primeros años.Las primeras publicaciones sobre investigaciones acerca de

Correspondencia: Luis Bravo-Valdivieso. Escuela de Psicología. Facultad de Ciencias Sociales. Pontificia Universidad Católica de Chile. Correo electrónico: abravov@uc.cl. 
las dificultades del aprendizaje escolar en los países sudamericanos analizados empezaron en la década de los años 50. Sin embargo, buscar información confiable sobre las primeras investigaciones ha sido difícil, debido a que la mayoría de ellas fueron publicadas en documentos de circulación limitada que no se encuentran con facilidad en las bibliotecas. Además muchas de ellas han sido discontinuadas y no ha habido intercambio entre los especialistas. Las principales revistas de psicología y de pedagogía aparecieron en las décadas siguientes.

La mayoría de las investigaciones iniciales fueron asociadas con trabajos neurológicos, pediátricos o de psicología clínica. Los estudios pedagógicos, con cierta base metodológica no se publicaban en revistas de educación. Esas revistas preferían hacer publicaciones teóricas o filosóficas. La preferencia por investigaciones clínicas tuvo como efecto el empleo de denominaciones de esa naturaleza, tales como " disfunción cerebral mínima”, "daño cerebral”, "hiperactividad", "dislexias" y "deficiencia mental", lo cual creó algunas confusiones entre los distintos especialistas, ya que no había intercambio de investigaciones que permitieran establecer patrones comunes de diagnóstico.

\section{Los primeros estudios clínicos}

Los primeros estudios clínicos sobre las Dificultades de Aprendizaje que hemos podido pesquisar fueron los de Quirós y Azcoaga en Argentina, de Olea en Santiago de Chile, de Mendilarhasu y Rebollo en Uruguay, de Sardi de Selle y Feldman en Venezuela, de Alarcón y Blumenfeld en Perú. Todos ellos aparecieron en las décadas de los años 50 y 60 . Tal vez el libro más conocido en nuestro medio fue "La Dislexia en la niñez" (1965) de Quirós, donde describe las dislexias como "una perturbación del lenguaje que perturba el aprendizaje y desarrollo de la lectura y escritura" (pág. 12). Según Quirós, la dislexia severa sería un tipo de afasia infantil, diferente del retraso en lectura y escritura producido por factores educacionales.

En Venezuela se crearon escuelas especiales para ciegos, sordos, mudos y otros "anormales" en 1912 (Tarnopol y Tarnopol, 1981). No tenemos información de investigaciones clínicas o publicaciones efectuadas en ellas.

En Chile, en 1923, en el Laboratorio Experimental del Instituto Pedagógico, el profesor Tirapegui adaptó para este país los tests de inteligencia de Binet y Terman y "prestó es204 pecial atención al tratamiento de niños con dificultades de aprendizaje" (Salas y Lizama, 2009, Pág. 93).

Posteriormente en Chile, en la década del sesenta, Olea y Moyano (1962) y Olea (1966, 1970) publicaron varios artículos en las revistas de Pediatría, sobre los aspectos clínicos de los niños disléxicos. Ellos asociaban las dislexias con la disfunción cerebral mínima y consideraban que provenía de deficiencias en la percepción y orientación espacial, y del desarrollo psicomotor. Desarrollaron dos tests destinados a evaluar el desarrollo psicomotor de los niños con dificultades para aprender a leer o escribir. Bravo (1968) publicó un artículo en la Revista chilena de Pediatría sobre las dificultades del aprendizaje escolar de la lectura, desde un punto de vista clínico y psicométrico, en grupos de alumnos que consultaban en el servicio de psiquiatría infantil del Hospital Calvo Mackenna de Santiago de Chile.

En 1970 M. Condemarín y M. Blomquist publicaron un exitoso libro dedicado a describir y diagnosticar las dislexias. En este período inicial, en Uruguay la investigación comenzó con los trabajos de Carbonell de Grompone y colaboradores (1965) quienes investigaron a los niños con dificultades para aprender a leer y escribir, desde el punto de vista del desarrollo de su orientación espacial. En 1975 Carbonell publicó otro artículo sobre las dificultades del aprendizaje lector y las dislexias. En ese mismo período varios especialistas crearon la Sociedad de Dislexia del Uruguay. Las investigaciones iniciales sobre las Dificultades del Aprendizaje culminaron con el Primer Congreso hispano Americano de lectura y escritura en Viña del Mar en 1971. La asistencia fue numerosa y se centró principalmente en los problemas de lectura y escritura en los niños de bajo nivel socio cultural. Es interesante mencionar que los trabajos eran más bien descriptivos que empíricos y que los autores no latinoamericanos más citados en las diferentes publicaciones fueron Ajuriaguerra y Zazzo de Francia, Piaget de Suiza, y Strauss, Myklebust y Cruickshank de los Estado Unidos. En Colombia, 1975 el Instituto Interamericano del Niño organizó un curso internacional, que tuvo numerosa asistencia, sobre Dificultades de Aprendizaje, en Bogotá y en 1983 se realizó otro Symposium en Medellín, presidido por William Cruickshank, que se centró en el tema de la disfunción cerebral infantil, el déficit atencional y la parálisis cerebral. 


\section{ALGUNAS EXPERIENCIAS EN DIVERSOS PAISES}

\section{La experiencia en Uruguay}

En 1972 se publicaron en Uruguay una serie de libros titulados "Estudios neuropsicológicos" donde se analizaba el problema de las dislexias, en trabajos de Mendilaharsu, quien con otros colaboradores elaboraron una batería de tests. Su objetivo fue investigar la inteligencia, la percepción visual, las habilidades espaciales y el lenguaje de los niños disléxicos. Entre sus resultados encontraron que los disléxicos tenían mayores dificultades en repetir oraciones orales y eran más lentos en procesar secuencias y ritmos. Asociaron las dislexias con las disfasias.

Las investigaciones sobre dislexia de evolución en Uruguay se han hecho principalmente dentro del ámbito neurológico y educativo. Hubo un cierto auge por estudiar e investigar sobre este tema en la década del sesenta, por iniciativa de destacadas figuras de nuestro medio como María Carbonell de Grompone, Carlos Bauzá, Elida Tuana y Magda Louzan entre otros.

La preocupación por el rendimiento escolar de los niños y en particular por conocer sobre los trastornos en lectura que presentaban algunos alumnos para los cuales se hacía necesario elaborar programas adecuados de intervención, llevó a que en 1962 se publicara en la revista "Archivos de Pediatría del Uruguay" un trabajo llamado "La dislexia de evolución: estudio de 35 casos personales" de Bauzá y colaboradores.

En este trabajo se definió la dislexia de evolución en términos operacionales como "una dificultad selectiva para el aprendizaje de la lectura, en presencia de un nivel mental adecuado para aprender a leer, o la incapacidad de sobrepasar un nivel inferior de lectura, lo que representa una detención en el proceso de adquisición de esta técnica" (Bauzá et al. 1962, p.310).

A partir de sus resultados plantearon la posibilidad de diagnosticar con anticipación la dislexia teniendo en cuenta determinadas características que pueden presentarse en los niños: no discriminar entre formas similares, inadecuada lateralización, desorientación espacial, dificultades para discriminar sonidos parecidos, retrasos en la motricidad, déficit en el lenguaje oral (dislalias, tartamudez y paragramatismo) o un vocabulario pobre.
Asimismo dentro de los síntomas que caracterizarían a la dislexia de evolución señalaban la confusión espacial de letras; confusión en el orden espacial de las sílabas y las palabras; adición u omisión de sonidos, sustitución u omisión de palabras; reducción en la velocidad lectora y pobre rendimiento en la escritura al dictado y espontánea.

En 1963 se publican las actas y trabajos del Primer Seminario sobre Dislexia, organizado por la Sociedad de Dislexia del Uruguay. Fundamentalmente se destaca la importancia de un diagnóstico temprano del trastorno lector y la relación entre lectura y escritura como un único proceso que lleva a que los niños disléxicos sean además disortográficos. (Carbonell de Grompone, Della Cella, 1963). Se planteó la relación de la dislexia con dificultades en la lateralidad, esquema corporal, desarrollo motriz, discriminación auditiva y visual, desarrollo del lenguaje oral y el cálculo, denominados "funciones básicas" (Carbonell de Grompone; Álvarez de Guadalupe; Louzán; Méndez de Brum y Tuana, 1963). Además se insistió en la importancia del método fonético para la enseñanza de la lectura en los niños disléxicos, dado lo inadecuado que resulta la enseñanza por el método global (Carbonell de Grompone, P. de Braslavsky, 1963).

En 1964 Carbonell de Grompone publicó un artículo sobre la organización espacial y su relación con la dislexia de evolución, donde evalúa la grafía y la reproducción de figuras geométricas sencillas en niños disléxicos. En sus resultados observa que los niños presentan una desorganización espacial significativa, lo que incidiría en su aprendizaje lector.

Por su parte Bay de Escobar (1965) publicó en un trabajo dirigido a los maestros preescolares para ayudarlos a la prevención de la dislexia de evolución. Considera que se debe tener en consideración los antecedentes familiares, la direccionalidad, las inversiones en las figuras, la reproducción de las estructuras rítmicas, la discriminación auditiva, la construcción de figuras y la nominación de objetos presentados en imágenes.

Louzán (1965) destacó que hay una pluralidad de síntomas que llevarían a diagnosticar la dislexia: la discordancia entre el $\mathrm{Cl}$ y el nivel lector alcanzado en lectura y escritura; nivel de lectura y escritura por debajo del promedio de la clase; diferencias con el rendimiento alcanzado en otras áreas; errores en lectura del tipo de 
rotaciones, inversiones, confusiones y sustituciones de letras; dificultades de orden simbólico; alteraciones en el lenguaje; dificultades de percepción, de esquema corporal, de motricidad, de memoria, en la capacidad de abstracción y generalización y desorientación espacial; trastornos neurológicos o emocionales; y antecedentes familiares. Criterios similares aparecen en los trabajos de Tuana $(1965,1967)$.

A partir de las publicaciones mencionadas, la mayor parte de los trabajos presentados hasta la fecha han vinculado la dislexia de evolución con diversos factores, tales como los trastornos en el habla, los problemas de lateralidad, y los déficits perceptivos y motrices (Romelli, 1986; Gabay Salinas, 2000); así como por factores emocionales (Gabay Salinas, 2000). También se asocia con problemas en la producción escrita como la disortografía (Carbonell de Grompone, 1975) o dificultades a nivel morfosintáctico (Zubiarre y Rodríguez 1979)

En cuanto a los subtipos de dislexia Tuana (1967) había identificado dos tipos de dislexia: la dislexia de percepción, que es cuando el niño realiza muy mal la copia, y la dislexia de audibilización, en que realiza mal el dictado y no es producto de un déficit auditivo. Pero la clasificación más frecuente, es la de Boder (1973), dada la amplia difusión e influencia que ha tenido en nuestro medio el test de lectura y ortografía de esta autora (Rebollo, 2004).

En un estudio realizado por Santini et. al (1977), en base a la metodología utilizada por Boder, indican que no se encontraron disléxicos disfonéticos en una muestra de niños uruguayos disléxicos, sólo se identificaron del subtipo diseidético. Marpeau (1996) trabajando con 10 disléxicos con edades de 7 a 10 años, en su mayoría repetidores de primero y segundo años de primaria, tampoco encontró disléxicos disfonéticos, y atribuyó estos resultados a un enmascaramiento de la fortaleza de la estrategia debido a diferencias idiomáticas y metodológicas.

En relación a estudios de tipo neurofisiológicos hemos encontrado el de Cibils et al. (1994) en el que con el objetivo de estudiar la participación de diferentes áreas de la corteza cerebral durante la lectura y escritura se estudió una población de 15 niños lectores normales y 15 disléxicos clasificados por el Test de Boder. Se estudiaron las variaciones porcentuales de la actividad alfa durante ejecución de pruebas de lectura y escritura seleccionadas. Los re- sultados mostraron un aumento de la actividad alfa (sincronización) durante la lectura y escritura en los disléxicos de tipo mixto. Diferentes comportamiento presentaron los otros subtipos; en comparación con los lectores normales. Los autores concluyeron que hay una participación diferente de las áreas de la corteza cerebral en niños normales y disléxicos durante la lectura y escritura. Es de destacar además el estudio Carbonell de Grompone (1975) aplicando la lectura de palabras del test de Boder, en el que encuentra que los niños con dificultades lectoras en castellano decodifican con gran lentitud pero no comenten demasiados errores.

A finales de los años 90 desde la Facultad de Psicología de la Universidad Católica del Uruguay se inició junto al equipo del Prof. Javier Marín de la Universidad de Murcia, una serie de trabajos de investigación en el área de la lectura y sus dificultades con el objetivo de poner en marcha la validación de un conjunto de pruebas que permitieran en nuestro medio evaluar la lectura y sus procesos implicados (Cuadro, 2002; Cuadro, 2005; Cuadro et., 2008; Trías y Cuadro, 2008; Marín et al, 2007a, 2007 b, 2007 c).

Se avanzó en la comprensión de las dificultades de los lectores retrasados en relación a los buenos lectores. (Cuadro, 2002, 2005, 2007a, 2007b; Carballo y Cuadro 2007; Cuadro y Marín 2008; Marín et al., 2008). Se diferenciaron diversos tipos de malos lectores y se compararon los resultados con otros estudios en castellano y en otros sistemas ortográficos (Cuadro, 2005; Cuadro y Marín 2007), y se desarrollaron programas de intervención (Cuadro, Trías, Castro, 2007).

Asimismo se iniciaron estudios en lectores sordos (Cuadro y Almansa, 2008), así como en niños no videntes lectores en Braille (Cuadro y Piñeyro, 2008).

\section{La experiencia en Argentina}

En Argentina, Azcoaga (1969) fue uno de los primeros y principales iniciadores de las investigaciones en este tema. Estudió las Dificultades del Aprendizaje desde el punto de vista clínico. El fue seguidor de los trabajos de Pavlov, Luria, Vigostsky y Ajuriaguerra, asociando las dislexias y el aprendizaje de la lectura con el nivel de desarrollo neuropsicológico infantil. Describe tres tipos de patologías que afectan los centros cerebrales superiores y originan diferentes tipos de dislexias. Azcoaga (1979) ha sido uno de los 
principales líderes en Sudamérica en la investigación neuropsicológica de las dificultades del desarrollo infantil. Fue fundador de la Sociedad Latinoamericana de Neuropsicología (SLAN) y de una Escuela profesional destinada a formar especialistas. Su influencia en Argentina ha sido muy relevante.

Posteriormente, las investigadoras argentinas Borzone de Manrique y Diuk (2001) investigaron las estrategias tempranas que emplean los niños para adquirir el conocimiento de las letras, lectura y escritura. Estudiaron a niños entre 6 y 10 años que asistían a los tres primeros años de escolaridad primaria. Los resultados mostraron el empleo de estrategias logográficas en los alumnos del primer año. En cambio en segundo y tercero utilizaron mayoritariamente una estrategia analítica. "Los errores en las escrituras mostraron la incidencia de la estructura fonológica en el uso de esta estrategia" (op.cit).

Posteriormente, Signorini y Borzone de Manrique (2003) profundizaron el empleo de las estrategias ortográficas y fonológicas en alumnos de segundo año básico y su eventual éxito en el aprendizaje. Los resultados mostraron "un efecto de complejidad ortográfica cuando la variable dependiente fue el tiempo de lectura" (op.cit). Sus resultados también confirman el peso que tienen las estrategias fonológicas, especialmente cuando los niños están aprendiendo a escribir. Ese mismo año, Diuk, Signorini y Borzone (2003a) investigaron el aprendizaje lector en niños de nivel socioeconómico bajo, de tercer año, comparándolos con niños de NSE medio de primer año. Sus resultados mostraron que los niños de nivel bajo presentan un patrón evolutivo cualitativamente similar al de los niños de nivel medio, aunque con retraso en su desarrollo. También encontraron que "los niños malos lectores compensan sus limitaciones de procesamiento fonológico apoyándose en su conocimiento ortográfico". Este grupo de investigación argentino ha sido uno de los de mayor productividad en los países del Cono Sur de Latinoamérica y se han concentrado principalmente en estudiar los procesos psicolingüísticos que determinan el aprendizaje de la lectura inicial.

\section{La experiencia en Perú}

En Perú, Reynaldo Alarcón publicó una extensa "Historia de la Psicología del Perú" (2000) en la Universidad Ricardo Palma. Algunas de las informaciones que colocamos a continuación han sido tomadas de este libro. La mayor parte de las investigaciones y publicaciones peruanas se realizaron en el contexto de la psicopedagogía y de la psicología educacional.

Según Alarcón una figura importante entre los promotores de esta disciplina fue Luis Miró Quesada, quien "siendo decano de la facultad de Filosofía y Letras de la Universidad de San Marcos fundó en 1925, un "Seminario de Pedagogía" en el cual investiga el valor psicológico y pedagógico de los tests..." (op.cit., pag 101). Este trabajo dio pie a nuevas investigaciones psicopedagógicas.

En 1942 apareció el primer número del "Boletín del Instituto Pedagógico nacional" donde figuran investigaciones "orientadas a estudiar la inteligencia de niños y adolescentes, para determinar el curso de su desarrollo, según la edad, la escolaridad y la influencia del nivel socio económico..."(Op.ci.).En 1955 se estableció en la Facultad de Educación de la Universidad de San Marcos un Instituto de Psicopedagogía, destinado a realizar investigaciones psicopedagógicas, dirigido por el famoso psicólogo Walter Blumfeld.

Según Alarcón el movimiento psicopedagógico peruano se caracterizó por el uso, casi exclusivo, de tests colectivos, siendo en su mayoría de procedencia norteamericana, sin embargo esta labor "fue antesala de la profesionalización de la psicología" (pág. 117).

Las primeras publicaciones peruanas de investigación en retardo escolar fueron asociadas con el retardo mental.

La principal investigadora peruana en este tema ha sido Alegría Majluf, quien desde 1970 ha publicado diversos trabajos en niños provenientes de las escuelas de sectores socioeconómicos deprivados en un libro titulado "Marginalidad, inteligencia y rendimiento escolar" (1993). Presenta 18 investigaciones. En sus investigaciones encontró que muchos niños con Dificultades de Aprendizaje presentaban desnutrición y deprivación crónica. Los maestros no conocían estrategias sociales y pedagógicas para ayudarlos y enseñarles con éxito. En 1976 ella siguió durante algunos años grupos de niños de clase media y clase baja usando las escalas de Piaget para evaluar la organización espacial, conservación y habilidades constructivas; encontró un claro progreso en ambos grupos. 
Sin embargo a los siete y medio años de edad los niños pertenecientes a clase media estaban significativamente más avanzados que los de nivel bajo en su desarrollo cognitivo.

En la década de los años ochenta la profesora fonoaudióloga Irma Villavicencio organizó en el colegio Palestra un congreso internacional sobre Psicopedagogía, que tuvo bastante concurrencia de otras naciones latinoamericanas, y en el cual se creó la Sociedad de Psicopedagogía Latinoamericana. Participaron en este encuentro psicólogos, neurólogos y educadores. Luego, fue invitado a Lima el investigador Frank Vellutino, quien dio varias conferencias a profesores especialistas en problemas de lectura.

En 1994 los psicólogos Torres y Luna reunieron diversas experiencias efectuadas con niños que presentaban dificultades de aprendizaje en un libro titulado "Problemas de Aprendizaje". Presentan resultados de programas correctivos, sistemas motivacionales y estrategias de intervención para mejorar habilidades escolares, como aritmética, ortografía y lectura. (Alarcón, 2000).

En la década de los años noventa y posteriormente, ha sido bastante importante, especialmente en Callao, el trabajo de la profesora Esther Velarde Consoli, de la Universidad Mayor de San Marcos de Lima, quien ha organizado cursos para formar maestros especialistas en educación especial para la Dificultades de Aprendizaje de la lectura y ha investigado la influencia de la conciencia fonológica en su aprendizaje.

\section{La experiencia en Paraguay}

La psicología en Paraguay también ha experimentado progresos en el estudio de las Dificultades de Aprendizaje, pero sus niveles de visibilidad todavía son escasos. García (2006) realizó una revisión de las publicaciones paraguayas en el área de la psicología, de 1960 al 2005. En forma genérica, enumeró las publicaciones en las áreas de la salud mental, psicología clínica, psicologías alternativas, psicoanálisis, psicología educacional, psicología del desarrollo y otros campos. Finalmente, discute algunas causas probables que podrían explicar la reducida productividad de la psicología paraguaya. Expresa que Palau Viladesau (1997) informaba años atrás que, a excepción del Instituto de Investigaciones en Ciencias de la Salud dependiente de la Facultad de Medi- cina y el Centro de Investigación perteneciente al Rectorado de la Universidad Nacional de Asunción, no existían en las universidades otras entidades para albergar investigación activa. A los mencionados agregaba el Centro de Estudios Sociales de la Universidad Católica que funcionó entre 1972 y 1975 . En su amplio listado de publicaciones e investigaciones no se mencionan trabajos en el área de la psicología educacional, psicopedagogía o neurociencias aplicadas a los trastornos del aprendizaje.

En 2007 apareció en Asunción un libro colectivo publicado por la Asociación Paraguaya de Neuropsicología, que aborda algunos temas relacionados con el aprendizaje de la lectura y de las matemáticas. Estos capítulos son resultado de investigaciones que siguen el modelo Fisiológico y Fisiopatológico del profesor argentino Juan E. Azcoaga, quien ha orientado al grupo de investigadores paraguayos.

\section{La experiencia en Colombia}

Dos vertientes fundamentaron el inicio del trabajo en trastornos del aprendizaje en Colombia: la psicopedagógica que influenció a psicólogos, pedagogos y al aparato educativo, apoyando incluso la toma de decisiones oficiales al respecto, y la que en principio podríamos denominar "médica" que influenció el ámbito de la salud, de manera especial a fonoaudiólogos, neurólogos y posteriormente neuropsicólogos.

La influencia de trabajos y publicaciones de otros países del cono sur específicamente de Chile y Argentina, se hizo notoria en Colombia en relación con el proceso enseñanza aprendizaje lector, y de manera específica en los niños con trastornos de lectura en las aulas y en las consultas de quienes apoyaban a aquellos infantes con algún tipo de dificultad. Los instrumentos y materiales de evaluación y enseñanza (Condemarín, M., Chadwick, M., Milicic, N. 1978) propuestos fueron ampliamente difundidos y usados, pero desafortunadamente no se realizó de ellos un seguimiento con interés investigativo que permitiera conocer el impacto real de sus aplicaciones para mejorar las prácticas educativas y solamente se pueden recoger narraciones de experiencias, siempre en términos positivos.

Más tarde, los trabajos de Alliende y Condemarín (1991) y Bravo Valdivieso (1985, 1999), enriquecidos con la recopilación de la investigación psicolingüística y de las neurociencias, 
en relación con el aprendizaje lectoescrito, marcaron su influencia en ese mismo terreno.

En los años ochenta, en el ámbito pedagógico, toman fuerza las posturas de algunos autores que niegan la existencia de los trastornos de aprendizaje, y reducen estos a problemas meramente pedagógicos o didácticos, y que en buena medida surgieron en respuesta a lo que se llamó la patologización de la educación.

Muy en consonancia con esta postura se empezó a criticar los llamados métodos tradicionales de enseñanza de la lectura, de manera especial el "de marcha", por centrarse en la apropiación del código y dejar en un segundo plano la comprensión lectora. Se hizo entonces una gran difusión de las publicaciones de Teberosky (1992) y Ferreiro y Teberosky (1989) en el ámbito educativo.

Desde la vertiente que se reconoció como médica, la influencia inicial desde finales de los años 60 fue la del grupo de Quirós, en el Museo Social en Buenos Aires, y debe relacionarse la gran influencia que en la naciente formación de fonoaudiólogos tuvo esta corriente con sus publicaciones. (Quirós y Della Cella, 1964, Quirós y Schrager, 1979, 1980) Ellas motivaron a profesionales del país a formarse con este grupo y a que se realizaran diversos cursos y congresos de problemas de aprendizaje en los años 70 . El desarrollo de los trabajos del grupo médico los llevó a la presentación de la hipótesis de Disociación propioceptiva vestibular y Escisión Vestíbulo-oculomotriz (Quirós, Schrager, 1980). Esta hipótesis es semejante a la que presenta en USA Jean Ayres (1973), desarrollando elementos específicos de evaluación e intervención, y tuvo un impacto que se mantiene hasta la actualidad en algunos ámbitos médicos y cuya práctica en relación con evaluación y tratamiento es campo de los profesionales de terapia ocupacional. Además ha permeado a la educación y es frecuente el uso por parte de los pedagogos de conocimientos de este tipo en su práctica diaria. Consideran que el apego a ese modelo da respuesta a sus necesidades profesionales cuando en sus aulas tienen niños con Trastornos de Aprendizaje, a pesar de haber también pronunciamientos que la consideran dentro de las Terapias controversiales (Kaplan y cols, 1993, Hoehn y Baumeister, 1994, Kamhi, 2004).

En la transición de los 70 a los 80 el modelo de comprensión y atención de Trastornos del Aprendizaje del grupo argentino de Azcoaga y colaboradores, empezó a conocerse en
Colombia y dominó la práctica de la evaluación e intervención de los Trastornos de Aprendizaje entre los fonoaudiólogos, y posteriormente en las escuelas. Sobre todo con la aplicación de sus conocimientos sobre el "Aprendizaje fisiológico" y el "Aprendizaje pedagógico", y la posibilidad de aplicar en la escuela lo que Azcoaga y su grupo de APINEP promulgaron como los pilares del aprendizaje, y la organización progresiva de analizadores complejos del lenguaje (Azcoaga 1969, 1979, Azcoaga y cols 1979, APINEP 1987).

La creación en la ciudad de Cali, del Instituto Lauretta Bender para trastornos del aprendizaje le dio gran empuje a la sensibilización hacia estos trastornos en el país. Se encargaron entre otras cosas de realizar congresos y cursos sobre ellos y la invitación a los grupos chilenos y argentinos reforzó el apego ya mencionado al trabajo de estos. El manejo de los niños en escuelas específicas con metodologías provenientes de la educación especial se empezó a generalizar en el país y de igual manera el Ministerio de Educación fortaleció las aulas de apoyo o especiales dentro de los colegios.

El interés por lo neuropsicológico en los Trastornos de Aprendizaje llegó de la mano del trabajo de Azcoaga, pero se desarrolló también por influencias desde las neurociencias cognitivas. En este sentido se han destacado los trabajos del grupo de Roselli y Ardila (1992). En relación con el déficit atencional, los trabajos de Pineda y cols. en Medellín (Pineda y cols. 1999, 2007, Palacio y cols, 2004) sobre la epidemiología de este trastorno en algunas regiones del país, así como sus investigaciones sobre la genética del mismo, han tenido gran resonancia. Sobre la difusión de material científico acerca de los trastornos del aprendizaje y además material de apoyo en evaluación e intervención de los mismos, se destacan a lo largo de las últimas dos décadas los Cursos Andinos de Trastornos del Aprendizaje de realización bienal, organizados por Eslava y Mejía del Instituto Colombiano de Neurociencias, y para los cuales han sido invitados investigadores principalmente del área Latinoamericana aunque también Europeos y Norteamericanos, y ha sido para Centroamérica y el Caribe, Colombia, Ecuador y Venezuela un punto de referencia en la temática. Un cuerpo de docentes de Chile (Milicic, N., Bravo Valdivieso, L., Aaron, A.M., Rosas, R., Allende F. y la siempre bien recordada Mabel Condemarín), así como de la Argentina (Azcoaga J.y Peña E.) y 
de México (Quintanar L., Solovieva Y.) han mantenido su presencia a lo largo de estos años con invaluables aportes para la formación y actualización de docentes y profesionales de la salud y la educación interesados en los Trastornos de Aprendizaje.

El grupo del Instituto Colombiano de Neurociencias en Bogotá ha generado materiales en Trastornos de Aprendizaje de los cuales vale la pena mencionar, la Guía para la Integración al Aula Regular del niño con dificultades (2003) y una TIC realizada para la Secretaría de Educación de Bogotá (Eslava-Cobos, 2008) destinada a la evaluación y seguimiento de los niños que inician escolaridad en la ciudad. También ha promovido la discusión acerca de los procesos de aprendizaje y los trastornos en los mismos buscando acercamientos e integración entre los sistemas de salud y educación para la eficiente atención de los niños, producto de los cuales hay diversas publicaciones alrededor del aprendizaje y sus trastornos (Mejía, L., Eslava,J. 1986, 2005) y sobre el Déficit Atencional (Eslava-Cobos J., Mejía L. 2008).

Mejía (1987) ha estudiado las relaciones del lenguaje con el aprendizaje lector, sosteniendo que los niños con trastornos o retardos del desarrollo del lenguaje presentan en la etapa escolar trastornos de aprendizaje como una manifestación más del problema lingüístico, en el cual los síntomas varían de acuerdo con las exigencias de la etapa de desarrollo.

También el tema de conciencia fonológica, su relación con el aprendizaje lector, el desarrollo del lenguaje en el aspecto fonológico sintáctico y la comprensión de la misma como un sistema funcional que comparte factores con los sistemas funcionales de lenguaje y lectura han sido desarrollados por Mejía y Eslava (Mejía, L. 2006, 2008, 2009 y Mejía \& Eslava, 2009).

La perspectiva educativa se debate aún entre continuar ignorando el area de los Trastornos del Aprendizaje por considerarlos nada más que una patologización de la educación (Mejia L., Eslava J, 2005), o reconocerlos y enfrentarlos como una realidad inocultable (Arias J 2003). Estos últimos, en general, han preferido encuadrar su perspectiva desde la óptica de los niños y niñas con necesidades educativas especiales (NEE), privilegiando los procesos de integración al aula regular (Cajiao 1992), aún cuando en los últimos años, esta perspectiva ha evolucionado - más allá de la integración - hacia procesos de inclusión (Florez R. y colaboradores 2006; Aldana L., Hernandez N., Mahecha M. 2006).

\section{La experiencia en Chile}

Desde el punto de vista histórico, el interés público en la Educación Especial para niños con déficits en su desarrollo comenzó en Chile en 1852 con la creación de una escuela para niños sordos. Posteriormente se creó una segunda escuela para niños ciegos, ambas en Santiago. En 1928 y 1929 se erigieron otras tres escuelas para niños con alteraciones de conducta. En 1945 fueron abiertas varias escuelas para niños con retardo mental, junto con algunos cursos especiales para ellos dentro de las escuelas corrientes. Un avance importante en Santiago fue la creación de la Clínica Psicopedagógica del Ministerio de Educación, en 1955, que tenía por objetivo el diagnóstico de los escolares con retardo en el aprendizaje.

En la década de 1960 se anexaron varias escuelas especiales a algunos servicios hospitalarios de pediatría, especialmente asociadas a los servicios de neurología y de psiquiatría infantil (Hospital Calvo Mackenna y Hospital Roberto del Río). Esta apertura se debió principalmente a la incorporación de los psicólogos al trabajo clínico y escolar y tuvo como consecuencia una buena integración profesional entre profesionales clínico y psicopedagógicos.

En la década de 1970 se crearon oficialmente los "grupos diferenciales" para niños con trastornos de aprendizaje y/o retardo mental leve, en numerosas escuelas públicas, sistema que impedía segregar a los alumnos con dificultades de la corriente principal del aprendizaje y los mantenía dentro de las escuelas comunes en las horas normales de clases.

Casi todas estas escuelas y cursos estaban orientadas o dirigidas por maestros especialistas, por fonoaudiólogos, por neurólogos y por psicólogos clínicos infantiles. Hasta la década de 1960 la mayoría de esos profesionales se habían formado en algún servicio hospitalario pediátrico, trabajando en equipos con médicos y otros especialistas. Al comienzo muchos de ellos eran auto formados.

Un avance fundamental para el desarrollo de la Educación Especial en Chile fue la creación en las universidades de cursos de postítulos dirigidos a maestros de educación básica y media para formarlos como profesores especialistas. Es así como en 1964 la Universidad de Chile creó el centro de formación de especialistas en deficiencia mental, y más tarde, en 1966 y 1970 abrió los postítulos en audición, lenguaje y trastornos de la visión. La Universidad Católica, por su parte, creo primero en 1968 un 
curso vespertino de postítulo en Trastornos del Aprendizaje y luego otro que otorgó el grado académico de Magíster en Educación Especial. Ambos cursos estaban destinados a maestros de educación básica o media, y educadoras de párvulos que atendían niños en los Jardines Infantiles y Kindergarten. Estos cursos los efectuaron inicialmente profesionales del Servicio de Psiquiatría Infantil del Hospital Calvo Mackenna. La especialización de estos profesionales y la creación de estos cursos universitarios produjeron numerosos estudios, publicaciones e investigaciones en este campo.

En 1975 se efectuó en la Universidad Católica de Santiago de Chile un Symposium propiciado por UNICEF llamado "EI niño limitado" en Chile, y en 1979 se realizó otro encuentro internacional, sobre "Los Niños con Dificultades de Aprendizaje", al cual asistieron especialistas de Argentina, Uruguay, Estados Unidos y, Colombia. También fue apoyado oficialmente y financiado por UNICEF. En este Symposium se hizo especial énfasis en las estrategias psicolingüísticas para el diagnóstico y tratamiento de las dificultades para el aprendizaje de la lectura y las dislexias. La discusión y el análisis de los trabajos presentados tuvieron un efecto importante en la modificación de las estrategias psicopedagógicas de la enseñanza del lenguaje escrito. Especialmente importante fue la intervención y presentación de los trabajos de Frank Vellutino, quien mostró investigaciones sobre la relación causal entre el aprendizaje de la lectura y el desarrollo del lenguaje. Hizo ver, en base a investigaciones empíricas comparativas entre disléxicos y niños lectores normales, que las dislexias no se originaban en trastornos de la percepción visual o de la psicomotricidad. Su participación produjo en Chile un cambio bastante radical en las estrategias pedagógicas de la enseñanza de la lectura.

En 1981 y en 1984 hubo en Santiago de Chile otros dos Simposios internacionales, uno sobre las dificultades en el aprendizaje de las matemáticas y el otro sobre Comprensión Lectora, también apoyados por UNICEF y la Universidad Católica de Chile.

En esa misma década, el gobierno chileno, preocupado por el problema del fracaso escolar temprano, llamó a un Seminario que reunió a profesores especialistas de todas las universidades que trabajaban este tema, además de otros especialistas del Ministerio de Educación pública. El trabajo de este Seminario duró varios meses y determinó las políticas públicas que se aplicarían en Chile en los años siguientes para ayudar a resolver los principales problemas escolares.

En la década de 1980 también se llevaron a cabo en Chile dos estudios experimentales, que tenían por objetivo ayudar a los alumnos a superar sus dificultades en el hogar y en las escuelas. Chadwick y Tarky entrenaron a un grupo de madres a ayudar a niños pre escolares para desarrollar el pensamiento lógico matemático. Ellas mostraron que es posible ayudar a los niños con bajo nivel socio económico en el aprendizaje escolar mediante un trabajo sistemático efectuado en el hogar. Milicic (1982) efectuó otra investigación tutorial en niños con alto riesgo de fracasar en lectura en primer año, comparándolos con un grupo de aprendizaje normal. Sus resultados mostraron un progreso de los niños como consecuencia del trabajo psicopedagógico de los tutores.

A partir de las dificultades detectadas por los niños con bajo rendimiento escolar, de las altas tasas de repetición con la consecuente deserción escolar, en los grupos más socialmente más vulnerables, diversos profesionales como Montenegro, Rodríguez, Lira y Orellana, desarrollaron programas de intervención temprana en los Jardines infantiles y en los hogares. También generaron instrumentos, que permitieran la detección temprana de trastornos en el desarrollo cognitivo.

En esa década también se observó en la educación chilena una fuerte influencia del modelo psicológico de Feuerstein sobre modificabilidad cognitiva, quien pone énfasis en desarrollar instrumentos para medir el potencial de aprendizaje, que la persona evaluada puede rendir en el momento de aplicar los test. Este modelo de evaluación de potencial de aprendizaje ( Learning Potencial Assessment Device, LPAD) ha sido aplicado a los niños en desventaja sociocultural y a niños con dificultades para aprender en diversos países. Los trabajos de investigación en este modelo más relevantes en Chile fueron los de Assael y Neumann (1989) y Morales (2003). Sin embargo, ellos no constituyen un cuerpo de investigación continuo que avale la validez de las intervenciones ampliamente utilizada en los contextos educativos con niños de diferentes déficits cognitivos.

Este modelo está fuertemente influenciado por un modelo constructivista, orientado no solo a rehabilitar sino que a generar nuevas 
estrategia que enriquezcan el funcionamiento cognitivo, buscando que los niños aprendan a aprender. La modificabilidad cognitiva surge de la experiencia de aprendizaje mediado, la que se realiza a través de un programa de intervención psicoeducativa. En el área de las neurociencias es importante señalar las investigaciones de Alberto Galaburda (1997, 2006) sobre las dislexias, un neurólogo chileno que trabaja en los Estados Unidos y descubrió primero importantes anomalías en la anatomía cerebral de los disléxicos. Especialmente debido a la influencia genética sobre el desarrollo temprano de las neuronas del área temporal del hemisferio cerebral izquierdo como efecto de la testosterona. Su trabajo ha tenido repercusión internacional sobre el origen de las dislexias. Lamentablemente no ha tenido un equipo de investigadores en Chile que sigan sus trabajos.

\section{Modificaciones en el paradigma psicopeda- gógico en la década de los ochenta}

En la década de los años ochenta la concepción de "educación especial" aumentó su énfasis en el "enfoque escolar" de las dificultades de aprendizaje, en la medida en que se formó un mayor número de maestros especialistas y hubo mayor interés en las autoridades públicas de educación por las dificultades escolares y preocupación por el bajo rendimiento en los aprendizajes de lectura y de cálculo. Junto con ello, disminuyó la atención de los niños con dificultades de aprendizaje en clínicas públicas, trasladándose el problema desde la clínica psicológica y neurológica a la psicopedagogía. Incluso hubo algunas personas que negaron la existencia de las dificultades específicas de aprendizaje y de las dislexias, atribuyéndolas todas a causas de deficiencias sociales escolares. Como consecuencia, Bravo (1990) planteó la diferencia entre "dificultades generales de aprendizaje" y "trastornos específicos del aprendizaje", atribuyendo estos últimos a alteraciones en el desarrollo neuropsicológico y los primeros a causas socio culturales o deficiencias metodológicas.

Así mismo esta hipótesis de la no existencia de las dislexias y de los trastornos específicos del aprendizaje, fue sostenida en Chile por algunos autores franceses que seguían a Mira Stamback, como Jossette Jolivet, atribuyéndolas solamente a orígenes socio económicos y culturales, lo cual influyó negativamente en muchos grupos que trabajaban con niños con necesidades educativas específicas. Su influencia, produjo un giro en las estrategias de enseñanza y se suprimieron en Chile muchos Grupos Diferenciales. Un fenómeno similar sucedió en Perú. Este cambio pedagógico no ha mostrado tener mayor beneficio para los niños y ahora se está modificando.

La hipótesis que las dificultades de aprendizaje tienen solamente un origen social o escolar ha sido desmentida por la gran cantidad de investigaciones en neurociencias que han aparecido en la última década que muestran diferencias significativas entre niños disléxicos y niños lectores normales. Los avances en neuropsicología infantil han venido a avalar la validez de las hipótesis, de las décadas de los años setenta, que tenían un enfoque de preferencia clínico, aunque no descartaban la influencia pedagógica. En la actualidad se ha originado cierta convergencia entre los aportes de la psicología, la pedagogía y la neurología, tanto en el campo de la investigación como en la práctica clínica. Las investigaciones internacionales, entre ellas las de Galaburda (1993) y Shaywitz (2008) han confirmado la existencia de un número de escolares que presentan alteraciones en el funcionamiento en algunas zonas cerebrales, que perturban el aprendizaje inicial de la lectura. Estas investigaciones se han efectuado principalmente con metodologías propias de las neurociencias, como ser las Imágenes producidas por la Resonancia Magnética nuclear, el EEG y los Potenciales Evocados y los estudios de anatomía patológica, las cuales no se aplican siempre en nuestros países sudamericanos. También se pueden ver las investigaciones de Habib 1997 y Paulesu 1996, que confirman la existencia de alteraciones en el funcionamiento cerebral en las dislexias, independientemente de las condiciones socio económicas y del C.I de los niños.

En Chile, Bravo (2004) publicó una exhaustiva revisión de esas investigaciones internacionales y su aplicación al campo pedagógico. Los resultados de ellas muestran que hay en todos los países un porcentaje de alumnos que tienen déficits en el desarrollo de algunos procesos cerebrales que demoran o interfieren su aprendizaje lector, por lo tanto en todas las escuelas primarias hay un grupo de niños que requiere de soluciones psicológicas individuales, pues no pueden aprender solamente con metodologías de enseñanza colectivas. 
Las investigaciones también han mostrado que además hay otros niños, con problemas menos específicos de aprendizaje, o llamados de aprendizaje lento, que pueden ser atendidos en grupos pequeños, en los cuales se les da una atención más focalizada a sus necesidades, de manera paralela a su escolaridad normal.

"La educación especial así concebida tiene por objetivo resolver los problemas a los niños que presentan alguna diversidad desde la perspectiva escolar. Son alumnos que no logran los niveles de aprendizaje esperados, debido, primariamente a interferencias originadas por algunos déficits de su desarrollo. Se caracteriza por asumir la "diversidad" de algunos niños, dentro del mismo sistema escolar, tratando de lograr la plena integración escolar de ellos. La intervención psicopedagógica especial tiene por objetivo darles la ayuda complementaria que les permita superar la segregación que los aísla de sus compañeros en el interior de las salas de clases." (Conferencia Universidad de La Serena: Luis Bravo V. "Psicopedagogía, Psicología Educacional y Educación Especial" 1998).

En Chile, también hubo numerosos trabajos en estas últimas décadas que se relacionan con el síndrome de déficit atencional, el que tiene alta comorbilidad con las dificultades de aprendizaje lector (Gorostegui 1997; Condemarín, Gorostegui y Milicic (2004); Lopez, Troncoso, Fostery Mesa (1998).

En relación a las variables familiares destacan los aportes de Chadwick y Scagliotti (1990), desde el punto de vista eco sistémico, y de Carrasco, en relación a las habilidades terapéuticas para atender familias con dificultades. Recientemente, Milicic, Mena, López de L. y Justiniano (2008) han planteado la necesidad de integrar la atención de los niños en redes escolares que incluyan la atención psicológica.

En la actualidad en Chile hay una vuelta al uso de los términos dislexia, discalculia y trastornos específicos del aprendizaje, en la medida que las investigaciones neuropsicológicas van dando cuenta de diferencias estructurales y funcionales en los cerebros de los niños disléxicos, en comparación con los niños que no presentan el trastorno.

Desde el punto de vista psicopedagógico E. Orellana (En prensa) ha escrito un libro que conceptualiza lo que es leer, aprender a leer, cuándo iniciar este aprendizaje y cómo enseñar a leer. En su libro se presentan investigaciones que avalan el Modelo Interactivo que propicia la autora, y se describen algunos instrumentos de evaluación escolar del lenguaje escrito.

Otro aporte destacado en el área de la atención y tratamiento de los niños con necesidades educativas especiales, ha realizado desde la cátedra de psiquiatría infantil de la Facultad de Psicología de la Universidad Católica y de la fundación Mírame, Amanda Céspedes, quien ha publicado un libro titulado Cerebro, Inteligencia y Emoción (2007) cuyo objetivo es ser un aporte de las neurociencias aplicadas a la educación.

\section{Los Tastornos de Aprendizaje y las diferencias socio culturales}

Uno de los principales problemas psicopedagógicos en Sudamérica es el efecto de la pobreza y carencia de educación en los sectores más pobres de la población y de las familias, lo cual afecta el desarrollo cognitivo y verbal de los alumnos que ingresan a las escuelas públicas. Este déficit impide que muchos niños aprendan a leer, escribir o calcular dentro de los plazos programados, y se configure un grupo muy numeroso de "escolares lentos" que con frecuencia son confundidos con retardados mentales leves. En consecuencia, uno de los primeros trabajos que hubo que efectuar fue crear o adaptar técnicas psicométricas de evaluación y diagnóstico que permitieran detectar a los alumnos con trastornos específicos de aprendizaje y distinguirlos de los niños cuyo rendimiento estaba originado en factores socio culturales o pedagógicos.

Los niños culturalmente deprimidos y que viven en pobreza son definidos en algunos países desarrollados como "minorías sub culturales", en comparación con la mayoría de alumnos que disponen de recursos económicos y culturales que les han permitido lograr un desarrollo cognitivo cuando ingresan a la escuela y una motivación suficiente para el aprendizaje escolar. En nuestros países sudamericanos los niños que viven en pobreza constituyen una mayoría, y esa pobreza no sólo afecta a los alumnos y a sus familias, sino también a la calidad de sus escuelas. Como consecuencia, el rendimiento escolar es insuficiente y muchas veces su lentitud para aprender se confunde con trastornos específicos del aprendizaje o dislexias.

Algunas investigaciones comparativas muestran que las habilidades verbales y fonológicas entre niños de escuelas públicas gratuitas y 
de escuela privadas pagadas tienen un desfase inicial desde Kindergarten y primer año básico, antes que las escuelas puedan intervenir en superar las diferencias (Bravo, 2009).

Como consecuencia de esta situación, un trabajo de investigación con los niños que presentan dificultades para aprender fue efectuar estudios diferenciales que permitieran determinar las áreas cognitivas o del lenguaje que afectaban el rendimiento en el grupo de alumnos que repetían curso en los primeros años básicos y que pertenecen a sectores culturalmente deprivados.

Sin embargo, las investigaciones en retardo lector y en dislexias en niños de bajo nivel sociocultural y económico también presentan problemas metodológicos severos debido a sus diferencias psicolingüísticas y familiares. Muchos de esos niños tienen dificultad cultural para el reconocimiento fonémico y semántico de los textos escritos, letras, sílabas y palabras, que van produciendo un retraso acumulativo del aprendizaje del lenguaje escrito, que se manifiesta con claridad a finales de la educación básica. Por otra parte, es difícil para los investigadores distinguir metodológicamente entre los niños con retardo lector severo provenientes de un NSE deficiente, entre aquellos cuyos déficits son causados por deficiencias culturales, de aquellos con una dislexia originada en anomalías neuropsicológicas o fonológicas, debido a insuficiencia en los instrumentos de diagnóstico para niños latinoamericano hispano parlantes.

Otro problema que se presenta para investigar o diagnosticar las dificultades de aprendizaje en alumnos de bajo NSE proviene de la norma - explícita o implícita- seguida por la mayor parte de los investigadores norteamericanos y europeos de exigir que las dislexias sean diagnosticadas solamente en niños con oportunidades socio culturales favorables, descartando como disléxicos aquellos que provienen de ambientes socioculturales deprivados (Chritchley, 1970).

Esta exclusión diagnóstica de muchos niños con deprivación sociocultural constituye un obstáculo severo para las investigaciones de las dificultades del aprendizaje de la mayoría los niños sudamericanos ya que forman el grupo mayoritario de la población escolar que también asiste a escuelas con severos déficits económicos y pedagógicos.

Una estrategia para superar esta dificultad fue efectuar una investigación comparativa entre niños con buen y bajo rendimiento escolar, pertenecientes a las mismas escuelas y aulas, equiparados por edad, $\mathrm{Cl}$, sexo y NSE, durante cuatro años.
El seguimiento mostró que las diferencias en el aprendizaje entre alumnos de bajo NSE no se explicaba por diferencias en los factores socio económicos. Las mayores diferencias en el aprendizaje estuvieron en las pruebas de conciencia fonológica, memoria de secuencia de letras que formaran una palabra, comprensión auditiva de una historia, y en abstracción verbal. Esos resultados mostraron que las diferencias socioeconómicas no aparecían ser la principal causa de las diferencias en el rendimiento entre los buenos y los malos lectores del grupo estudiado (Bravo y col., 1995).

El resultado de este seguimiento de cuatro años puede asociarse con otro estudio de seguimiento entre primero y cuarto año básico de 227 alumnos recién ingresados al primer año. El estudio consistió en determinar cuáles eran las principales variables predictivas del rendimiento en lectura a fines del cuarto año, comparando también los buenos y malos lectores. Todos los sujetos pertenecían a un nivel socioeconómico bajo. Los resultados de la matriz de correlaciones entre las pruebas iniciales y la lectura final y de regresión step-wise sobre la lectura, mostraron que las variables con mayor predictividad del lenguaje escrito fueron el conocimiento inicial de las letras, el reconocimiento de algunas palabras escritas y la conciencia fonológica, todos ellos evaluados al momento de ingresar a primer año (Bravo, Villalón y Orellana, 2006). Las diferencias en lectura entre los niños con buen y mal rendimiento inicial al ingresar al primer año se mantuvieron hasta fines de cuarto año y no se explicaron por sus diferencias socio económicas.

\section{CONCLUSIONES}

Se podría plantear como conclusión de este breve resumen, que la investigación en educación especial en Trastornos del Aprendizaje escolar en Sud América parte principalmente, de dos ámbitos.

El primero ligado a la educación, que hace emerger la relevancia del tema del fracaso escolar y la deserción. Dentro de esta perspectiva los trastornos específicos del aprendizaje, especialmente las dislexias, surgen como una de las causales más directas y aparecen con frecuencia asociados con factores socio económicos y emocionales familiares.

El segundo aparece ligado al ámbito de la salud. Surge de los servicios de psiquiatría y neurología de los hospitales de niños, los 
cuales se ven inundados de consultas de niños con hiperkinesia, impulsividad, dificultades en la atención y concentración y el aprendizaje de la lectura. Los especialistas de estos servicios abordan el tema desde la perspectiva de la descripción de los síndromes que tiene la población consultante, y efectúan investigaciones acerca de la efectividad del tratamiento medicamentoso y la comorbilidad de los trastornos de aprendizaje con el déficit atencional.

En ambos grupos aparecen, en los distintos países latinoamericanos estudiados, publicaciones de diversos profesionales, en su mayoría psicólogos, neurólogos, psiquiatras infantiles y fonoaudiólogos, que se insertan en los medios universitarios, y trabajan en la formación inicial de los nuevos profesionales.

Algunos de los temas en los que se desarrolló la investigación, en el período inicial de la década de los años cincuenta y sesenta, fueron: prevalencia de los Trastornos de Aprendizaje en la población escolar, construcción y estandarización de instrumentos nacionales o adaptación de test extranjeros, orientados a la evaluación de los problemas de aprendizaje, en áreas como la lectura, la escritura y el cálculo, estrategias de intervención y elaboración de programas y material orientado a la rehabilitación de los trastornos. Sin embargo, no todos ellos tuvieron el éxito deseado debido a las diferencias de idiomas.

Posteriormente se han hecho análisis de algunas estrategias de asesoría psicoeducativa a los padres con el objeto de fortalecer las competencias parentales necesarias para lograr un buen abordaje de las necesidades educativas de los niños, cuyas conductas disruptivas y falta de logros impactan negativamente el contexto familiar y escolar.

Estas investigaciones han tenido un impacto importante, aunque no suficiente en la generación de políticas públicas orientada a la atención de los niños con necesidades educativas especiales. El primer gran problema ha sido entregar información lo más rigurosa posible a las autoridades que toman las decisiones y proponer estrategias de atención, como por ejemplo la integración de niños con necesidades educativas especiales, la evaluación diferencial, la generación de escuelas de lenguaje y, los gabinetes psicopedagógicos al interior de las escuelas. En la última década han aumentado las investigaciones en neurociencias, ligadas a los programas de Magíster y Doctorado en las universidades sudamericanas, y que han abierto un nuevo camino al estudio de los Trastornos de Aprendizaje, tanto desde el punto de vista clínico, como educacional. En este ámbito la investigación latinoamericana se acerca a los patrones que encontramos en los principales centros internacionales de países más avanzados en investigar estos temas.

\section{REFERENCIAS}

Alarcón Reynaldo (2000). Historia de la Psicología en el Perú. Lima. Universidad Ricardo Palma.

Aldana, L., Hernández, N. y Mahecha, M. (2006). De diagnósticos clínicos a procesos educativos-pedagógicos. En: Integración escolar. Aprender desde la diferencia. Red Aprender desde la diferencia. Red de Maestros Integradores. Universidad Nacional de Colombia.

Alliende F. y Condemarín M. (1982) La lectura: Teoría, evaluación y desarrollo. Santiago de Chile. Andrés Bello.

Alliende, F. , Condemarín, M. y Milicic N. (1991). Prueba de Comprensión Lingûística Progresiva CLP. Formas Paralelas. Santiago de Chile, Ediciones U. Católica de Chile.

Arias, J. (2003). Dificultades de aprendizaje. Universidad Pedagógica Nacional. Facultad de Ciencias y Educación. Bogotá.

Armele,M., Barriocanal L., Ferreira M.E., Ojeda M, Rumich D. y Scavone J. (2007) Neuropsicología, aprendizaje y ciencia. Asunción. Asociación Paraguaya de Neuropsicología.

APINEP. (1987). Psicología, Lenguaje, Aprendizaje. Buenos Aires. Ediciones pedagógicas.

Ayres, J. (1973). Sensory Integration and learning disorders. Los Ángeles, CA: Western Psychological Services.

Azcoaga J.E. (1969) ¿Qué es la dislexia escolar? Rosario: Editorial Biblioteca.

Azcoaga, JE., Derman, B., Iglesias, P.A. (1979). Alteraciones del aprendizaje Escolar. Diagnóstico, fisiopatología, tratamiento. Buenos Aires: Paidós.

Azcoaga J.E (1979) Aprendizaje fisiológico y aprendizaje pedagógico. Buenos Aires: El Ateneo.

Bauza, C., Carbonell de Grompone, M., Drets, M. y Escuder, E (1962). La dislexia de evolución: estudio de 35 casos personales. Archivo de pediatría del Uruguay: 34: 43-53, 112-123.

Bay de Escobar, M. (1965). Posibilidades de un despistaje de predisléxicos en clases de jardinera y jardines de infantes. Boletín del Instituto Interamericano del Niño, julio: 497-502.

Berdicewski O. y Milicic N. (1978) Manual del Test de Funciones Básicas. Santiago de Chile: Editorial Galdoc.

Berdicewski O, Milicic N. y Orellana E. (1983) Elaboración de normas para la Prueba de Dislexia Específica de Condemarín-Blomquist. Santiago de Chile. Universidad Católica.

Borzone de Manrique y Signorini A ( 1994) Phonological awareness, spelling and reading habilities in Spanishspeaking children. British Journal of Educational Psychology, 64,429-439.

Borzone de Manrique y Signorini A. (1998) Emergent writing forms in Spanish. Reading and writing: An interdisciplinary journal, 10, 499-577. 
Borzone de Manrique A.M. y Diuk B. (2001) El aprendizaje de la escritura en español: Estudio comparativo entre niños de distinta procedencia social. Revista de Psicología y Ciencias afines, 18,35-63.

Bralic, S. Haeussler, M., Lira, M., Montenegro, H. y Rodriguez, S. (1979 ) Estimulacion temprana: Unicef Santiago Chile.

Bralic, S y Rodríguez, S (1981) Desarrollo psicomotor en niños chilenos de nivel socioeconómico bajo provenientes de distintos sectores geográficos y étnicos. CEDEP.

Bravo L. ( 1990) Psicología de las Dificultades del aprendizaje escolar. Santiago de Chile: Editorial Universitaria ( $8^{a}$ edición 2009).

Bravo L. (1968) Trastornos del desarrollo psicológico en niños con daño cerebral mínimo. Revista Chilena de Pediatría, 39,369-380.

Bravo L. (1995) A four year follow-up study of low socioeconomic status Latinamerican children with reading difficulties. International Journal of Disability, Development and Education, 42,189-202.

Bravo Valdivieso, L. (1985). Dislexia y retraso lector. Madrid: Santillana.

Bravo L (2004) Lenguaje Escrito y Dislexias. Santiago de Chile: Ediciones de la Universidad Católica.

Bravo, L., Villalón,M. y Orellana, E. (2006) Diferencias en la predictividad de la lectura entre primero y cuarto años básicos. Psykhé, 15, 3-12.

Bravo, L., Villalón, M. y Orellana, E. (2006) Predictibilidad del rendimiento en la lectura : Una investigación de seguimiento entre primer y tercer año. Revista Latinoamericana de Psicología, 38, 9-20.

Bravo L. (2006) Lectura Inicial y Psicología Cognitiva. Santiago de Chile: Ediciones de la Universidad Católica.

Bravo, L .(1997) Una convergencia entre las neurociencias y la psicología cognitiva. Los estudios sobre el lenguaje y las dislexias. Psykhé 6, 23-34.

Bravo, L., Bermeosolo, J. ,Pinto, A. y Oyarzo E. (1996) Seguimiento de niños con retraso lector severo. Infancia y aprendizaje, 76, 3-12.

Cajiao, F. (1992). Fronteras de la normalidad: la integración al aula regular del niño con Trastornos del Aprendizaje. I Curso Andino de Trastornos del Aprendizaje.Validez de la evaluación del potencial de aprendizaje Psycothema 2, 217 - 221.

Carballo, M.; Cuadro, A. (2007). La velocidad de nominación en el déficit lector. Montevideo. Sociedad de Dislexia del Uruguay.

Carbonell de Grompone, M., Alvarez de Guadalupe, E., Della Cella, M. y Tuana, E. (1965). Actas y trabajos del I Seminario sobre Dislexia. Sociedad de Dislexia del Uruguay.

Carbonell de Grompone, M. (1975). Colonianismo y dislexia. En El lenguaje lectoescrito y sus problemas. Buenos Aires: Editorial Médica Panamericana.

Carrasco, E. (2003) Decálogo sobre lo mínimo que debe saber un terapeuta para trabajar con niños. De familias y terapias 11,13-19.

Céspedes, A . (2007) Cerebro Inteligencia y Emoción. Santiago de Chile. Fundación Mirame.

Condemarín ,M .; Gorostegui .,M.E.y Milicic , N.(2004) Déficit atencional. Santiago Chile: Editorial Planeta.
Condemarín M. y Blomquist M (1970) . La Dislexia. Manual de Lectura correctiva. Santiago de Chile: Editorial Universitaria.

Condemarín, M., Chadwick, M., Milicic, N. (1978). Madurez escolar. Santiago de Chile: Editorial Andrés Bello.

Cibils, D. et al (1994). Variaciones topográficas en la actividad alfa durante pruebas de lectoescritura en niños normales y en disléxicos, Revista Anales de Neuropediatría Latinoamericana. (Montevideo), 4, 2, 33-36.

Cuadro, A. (2001). Caracterización de buenos lectores en relación a los malos lectores en lengua castellana. Aprender un derecho de todos. Montevideo. Sociedad de Dislexia del Uruguay.

Cuadro, A. (2005) Caracterización de los lectores retrasados en relación a los buenos lectores en niños uruguayos. Tesis Doctoral. Universidad de Murcia. España.

Cuadro, A. (2006) Caracterización de los lectores retrasados. UBA. Buenos Aires.

Cuadro, A. (2007a). Caracterización de los lectores retrasados en español. Montevideo. Sociedad de Dislexia del Uruguay.

Cuadro, A. (2007b). Los trastornos en el aprendizaje de la lectura: la dislexia evolutiva. Montevideo. Sociedad de Dislexia del Uruguay.

Cuadro, A.; Trías, D.; Castro, C. (2007). Ayudando a Futuros Lectores. Montevideo: Prensa-Medica.

Cuadro, A.; Navarrete, I. (2007) Estilos atribucionales causales y rendimiento académico. Buenos Aires. UBA.

Cuadro, A. y Marín J. (2007) Los subtipos de dislexia en español. Revista Ciencias Psicológicas. V 1, 2, 133-148.

Cuadro, A. y Almanza, X. (2008) (remitido) Caracterización de los lectores sordos: un estudio comparativo con los lectores normales oyentes. Buenos Aires. UBA.

Cuadro, A., Piñeyro, V. (2008) (remitido) Caracterización de los lectores Braille: un estudio comparativo con buenos lectores videntes. Buenos Aires. UBA

Cuadro, A. y Marin, J. (2008) Reading Dysability Subtipos and Reading. XXX Internacional Neuropsychological Society Mid-Year Meeting. Buenos Aires.

Cuadro, A., Costa, D. Trias, D. y Ponce de León, P. (En prensa). Evaluación del Nivel Lector: Test de Eficacia Lectora Marin y Carrillo. Montevideo: Prensa Médica.

Chadwick, M y Scagliotti, J. (1990) Aproximación del enfoque sistémico a los trastornos del aprendizaje . Lectura y vida, 2, 17-25.

Chadwick, M., y Scagliotti, J. (1994) los trastornos de aprendizaje : Rol de la familia y la escuela . Revista de educación $N^{\circ}$ 223. Santiago Chile.

Diuk B., Signorini, A., Borzona, A.M. Las estrategias tempranas de lectura de palabras en niños de $1^{\circ}$ a $3^{\circ}$ año de educación general básica: Un estudio comparativo entre niños procedentes de distintos sectores sociales. Psykhé, 12, 51-62.

Eslava-Cobos, J. (2008.) Conociendo a los niños y las niñas, escribimos y planeamos el desarrollo. Bogotá: Instituto Colombiano de Neurociencias.

Eslava-Cobos J. y Mejía L.(2008) "Tres mil cuatrocientos ochenta millones (Editorial)". Acta Neurológica Colombiana; 24 (2): 49-52.

Eslava-Cobos J. (Ed. Invitado), Solovieva Y. (compiladora). (2008) Número monográfico de Neuropsicología Infantil. Acta Neurológica Colombiana; 24 (S2:1). 
Eslava Cobos, J., Mejía de Eslava, L., Quintanar, L. y Solovieva, Y. (2008). Los trastornos del Aprendizaje: perspectivas neuropsicológicas. Bogotá: Editorial Magisterio.

Eslava-Cobos J. y Ricaurte J. ( 2007) "Integración al aula: una estrategia para la intervención y evaluación guiada a través del uso de nuevas tecnologías de intervención (TICs)." Psykhe;16 (2): 69-84.

Eslava-Cobos J., Ricaurte J. (2006) "Integración al aula: un encuentro de saberes en ambientes de aprendizaje y evaluación" en IDEP (Ed.). Ambientes de aprendizaje y evaluación interlocutiva. Editorial Idepentiente,: 139-186

Eslava-Cobos, J. y Ricaurte, J. (2000) "La investigación como herramienta de capacitación y de trabajo". Psykhe 9(1): 27-37.

Eslava, J. y Mejía, L. (1985). Asimetría Cerebral y trastornos de aprendizaje. En: Asimetría Cerebral Funcional. Patricia Montañés., Asociación Col. de Neuropsicología, Bogotá. 133-45.

Feldman N. y Feldman M. (1981). Reading and learning disabilities in Venezuela. En: L. Tarnopol y M. Tarnopol (Eds) Comparative Reading and Learning Dificulties (pp 497-518) Massachusetts. Toronto: Lexington Books.

Feuerstein, R.; Rand, Y., Hoffman, M.B. y Millar, R. (1979) The dynamic assessment of retarded performers. University Baltimore: Park Press.

Ferreiro, E., Teberosky, A. (1989). Los sistemas de escritura en el desarrollo del niño. México: Siglo XXI.

Flórez, R., Moreno, M. y cols (2006). Una aproximación conceptual al proceso de integración escolar. En: Integración escolar. Aprender desde la diferencia. Red Aprender desde la diferencia. Red de Maestros Integradores. Secretaría de Educación Distrital y Universidad Nacional de Colombia.

Fundar (2002) Niños con necesidades educativas especiales como enfrentar el trabajo en el aula. Santiago de Chile. Fundacion Educacional Arauco.

Galaburda, A. (1993) Dislexia and Development. Neurological aspectos of extra-ordinary brains. Cambrige. Harvard University Press.

Galaburda, A. (2006) La dislexia del desarrollo: gen, cerebro y cognición. Psykhé, 15, 3-12.

García, J.E. (2006) Publicaciones paraguayas en el área de la Psicología: 1960-2000. Revista Latinoamericana de Psicología, 38, 149-167.

Gorostegui, M. E. (1997). Síndrome de déficit atencional con hiperactividad: Estilo cognitivo y rendimiento escolar. Psykhe 6, 63-70.

Habib, M. (1997). Dyslexie: Le cerveau singulier. Marseille. Solal.

Hallahan, D. y Keogh, B. (2001) Research and global perspectives in learning disabilities. London. Lawrence Erlbaum Ass.

Hoehn, T. P., Baumeister, A. A. (1994). A critique of the application of sensory integration therapy to children with learning disabilities. Journal of Learning Disabilities. 27(6) : 338-50.

Kamhi, A.G. (2004). A meme's view of speech language pathology. Language, speech and hearing services. 35(2): 105-11.

Kaplan, B.J., Polatajko, H.J., Wilson, B.N. y Faris, P. D. (1993). Reexamination of sensory integration treatment: a combination of two efficacy studies. Journal of Learning Disabilities. 26 (5): 342-7.

Louzan, M. (1965). Diagnóstico Global del retardo en lectura. Boletín del IIN. 155, 776-784 .Montevideo, Uruguay.

Lira, M. I. (1990) Manual de estimulación del segundo año de vida. Santiago de Chile: Editorial Galdoc.

Louzan, M. (1965). Lateralidad, Estructuración Espacial y Dislexia. Actas y Trabajos del I Seminario sobre Dislexia en el Uruguay. 110-122.

Majluf A. (1993) Marginalidad, inteligencia y rendimiento escolar. Lima: Editorial Brandon.

Marín, J., Aveledo, F., Pagán, A. P. y Cuadro, A. (2007ª). ¿Por qué es más complicado distinguir entre "berenjena" y "berengena" que entre "escabeche" y "escaveche"? AESLA. Murcia.

Marín, J., Pagán, A. P., Cuadro, A. y Aveledo, F. (2007b). Adquisición de Información ortográfica y mecanismos de recodificación fonológica: nuevas implicaciones para el modelo de autoaprendizaje (Share, 1995). AESLA. Murcia.

Marín, J.; Cuadro, A. Pagán A. (2007c) Orthographic Lexicon and ReadingAbility. Rev. Ciencias Psicológicas. 1, 15-26.

Marin, J., Pagán, A, y Cuadro, A. (2008) ERP. Correlates of Phonological Ambiguity in Spanish Orthography. $X X X$ Internacional Neuropsychological Society Mid-Year Meeting. Buenos Aires.

Mejia L. y Eslava-Cobos, J. (2005). "Los Trastornos del Aprendizaje: patologizando la educación o reconociendo realidades?". Revista Internacional Magisterio;15:16-20

Mejía L., Eslava-Cobos, J. y Ricaurte J. (2003) "Guia para la integración al aula regular del niño con dificultades". Bogotá: Santillana.

Mejía de Eslava, L (2009a) Conciencia Fonológica como predictor del aprendizaje lector. En: Dificultades en el proceso lectoescritor. Quintanar.

Mejía de Eslava, L. (2009b). Un acercamiento a la comprensión de la conciencia fonológica desde los enfoques neurofisiológico e histórico cultural. En: La perspectiva histórico - cultural y la neurofisiología. En: Feld, V y Eslava-Cobos, J. Editores. Buenos Aires: Noveduc. Capítulo 6. 127 - 38.

Mejía de Eslava, L. y Eslava Cobos, J. (2008). Conciencia fonológica y aprendizaje lector. Acta neurológica Colombiana 24 (2) Suplemento (2:1): S55 - S63.

Mejía de Eslava, L. (2006). Conciencia Fonológica y Aprendizaje lector. Neuropediatría. Revisiones 4 (1) :1-20.

Mejía de Eslava, L. (2006) .Presentación de caso clínico. Neuropediatría. Revisiones. 4 (1) : 21-23.

Mejía de Eslava, L. y Eslava-Cobos, J. (2005). Los trastornos del aprendizaje: ¿patologizando la educación?, o reconociendo realidades. Magisterio, 15: 16-20.

Mejía, L. (1992) Dislexia- Disgrafia. En: Neuropsicología Infantil. Rosselli, M., Ardila, A. Asociación Colombiana de Neuropsicología, Bogotá. 179-99.

Mejía, L. y Eslava, J. (1.989) Developmental and dissolution of language: a Neurophysiological Perspective. En: Brain organization of language and cognitive Processes. Ardila, A., Ostrosky-Solis, F, Plenum Press, New York.85- 102.

Mejía de Eslava, L. (1.987) Trastornos de Aprendizaje como secuela de Retardos del lenguaje. Facultad de Educación Preescolar. Bucaramanga. 3:77-81. 
Mejía, L. y Eslava, J. (1986). latrogenia en el aprendizaje. Infancia, 1, 3, 20-25.

Mendilarhasu C. (1972). Estudio de la Dislexia de evolución. Acta Neurológica Latinoamericana,18, 299-317.

Mendilarhasu C.(1981). Estudios Neuropsicológicos. Montevideo: Editorial Delta.

Milicic, N. (1983). Un Programa de Tutoría para la enseñanza de la lectura inicial. Lectura y Vida, 4,15.24.

Milicic N. y Schmidt S. (1978) Test de Pre cálculo. Santiago de Chile: Galdoc.

Milicic, N:, Mena, I., López de L.S., y Justiniano, S. (2008). Clínica Escolar. Prevención y atención en redes. Santiago de Chile: Editorial de la Universidad Católica.

Montenegro, H., Bralic, S., Edwards, S., Izquierdo, T. y Maltes, S. (1983). Salud Mental Escolar: estandarizacion del cuestionario de Inventario de problemas conductuales y Destrezas sociales de T. Achenbach. UNICEF: Santiago de Chile.

Morales (2003). El cambio cognitivo en el niño de aprendizaje lento: Santiago de Chile. Universidad Católica. U. Silva Henríquez.

Olea R. (1966). Daño cerebral mínimo. Revista Chilena de Neuropsiquiatría, 5,34-38.

Olea R. (1970). Disfunción cerebral mínima , daño o déficit cerebral del niño. Revista Chilena de Pediatría, 41,318323.

Olea R. y Moyano, H.S. (1962). Acerca de las bases neurológicas de la dislexia de evolución. Fonoaudiología 8.

Orellana, E. (1994). Una experiencia con Lenguaje Escrito en el nivel pre-escolar. Boletín de Investigación Educacional, 9,118- 133 .

Orellana, E. (1995). Instrumento de Evaluación ELEA. Fundamentos teóricos y análisis estadísticos. Boletín de Investigación Educacional, 10, 163-180.

Orellana E. (en prensa) Psicología de la enseñanza del lenguaje escrito. Santiago de Chile: Editorial LOM.

Palacio, J.D., Castellanos, F., Pineda, D.A., Lopera, F., Srcos-Burgos, M., Quiros, Y. y cols. (2004). Attention deficit/ hyperactivity disorder and comorbidities in 18 paisa Colombian multigerational families. J. Am Academy Child Adolescence psychiatry. 43: 1506-15.

Palau Viladesau, T. (1997). La investigación en Ciencias Sociales y de la Conducta en el ámbito universitario. En J. G. Britos (Ed.), 1er. Congreso Universitario de Psicología. Compilación de ponencias (pp. 62-67). Asunción: Universidad Nacional de Asunción.

Paulesu, E., Frith U., Snowling, M., Gallagher, A., y col. (1996) Is developmental Dyslexia a disconnection syndrome? Evidence from PET scanning. Brain, 119, 143-157.

Pineda, D.A., Ardila, A., Roselli, M., Arias, B., Henao, G., Gómez, L. F., y cols. (1999). Prevalence of Attention Deficit/ hyperactivity disorders symptoms in 4 to 17 years old children in the general population. Journal of Abnormal Child psychology. 27: 455-62.

Pineda, D.A., Palacio, L.G., Puerta, I.C., Merchán, V., Arango, C.P., Galvis, A.Y., Gómez, M., Aguirre, D.C., Lopera, F. y Arcos-Burgos, M. (2007). Environmental influences that affect attention deficit/hyperactivity disorder: study of a genetic isolate. European Child adolescence Psychiatry. 16: 337-46.
Quirós, J. B. (1968) Dislexia y Lenguaje. Dislexia Escolar. Instituto Interamericano del Niño. (pp 40-45) O.E.A. Montevideo.

Quirós, J.B (1959) La Dislexia como síntoma y como síndrome. Acta neuropsiquiátrica argentina, 5,178-193.

Quirós J.B y Della Cella (1965) La Dislexia en la niñez. Buenos Aires. Paidós.

Quirós, J.B. y Schrager, O. L. (1979). Lenguaje, Aprendizaje y Psicomotricidad. Buenos Aires. Panamericana.

Quirós, J. B., Schrager, O. L. (1980). Fundamentos neuropsicológicos en las discapacidades de aprendizaje. Buenos Aires: Panamericana.

Rebollo, M. (2004). Dificultades del Aprendizaje. Montevideo: Prensa Médica Latinoamericana.

Rosselli, M. y Ardila A. (1992). Neuropsicología Infantil. Asociación Colombiana de Neuropsicología, Bogotá. Págs. 179-99.

Rodríguez, S., Arancibia, V. y Undurraga, C. (1990). EEDP: Escala de evaluación del desarrollo psicomotor de 0-24 meses. Santiago de Chile: Editorial Galdoc.

Salas G. y Lizama E. (2009). Historia de la Psicología en Chile. Editorial Universidad de La Serena.

Sardi de Selle M. y Feldman N. (1971). La imagen de sí mismo en el niños con dificultades de aprendizaje y su proyección en el medio escolar. Revista Niños, 4, 4-15.

Santini de Souto, H. (1977). Tipos de dislexia. Neuropediatría Latinoamericana, 2, 41-48.

Shaywitz, S. y Shaywitz, B. (2008). Paying attention to reading: The neurobiology of reading and dyslexia. Developmental Psychopathology, 20,1329-1349.

Signorini, A. y Borzone de Manrique, A. M. (2003). Aprendizaje de la escritura en español. Predominio de las estrategias fonológicas. Revista de Psicología y Ciencias afines, 20, 5-30.

Smythe, I., Everattt ,J. y Salter, R. (2004). International book of Dyslexia. Chichester. John Wiley and sons. Ltd.

Tarnopol, L. y Tarnopol, M., (1981). Comparative reading and Learning Difficulties. Toronto: Lexington Books.

Tuana, E., Carbonel, M. y Lluch, E. (1980). Diez años de investigación ortográfica. Lectura y Vida. 1,16-19.

Tuana, E. (1965). Diagnóstico analítico de las dificultades en lectura. Boletín de IIN. Montevideo, №155, pp.785-796.

Tuana, E. (1967). Dificultades en el aprendizaje de la lectura y escritura. Boletín de la Inspección Nacional de Escuelas de Práctica. Montevideo, 11,1-12.

Teberosky, A. (1998). La lengua que escriben los niños pequeños. Reflexiones sobre una situación de aprendizaje. Buenos Aires: Editorial Aique.

Trias. D. y Cuadro, A (2008) (Remitido). Desarrollo de la conciencia fonémica: evaluación y seguimiento de un programa de intervención. Rev. Sociedad de Neuropsicología Argentina.

Yudelevich, E. (2003). Decálogo sobre la familia con uno o más, miembros afectados por el síndrome de déficit de atención , ya sea con o sin hiperactividad. De familias y terapias, 11, 25- 31.

Zubiarre, M. y Rodríguez, L. (1979). La comunicación escrita en el niño disléxico. Revista APPIA. Montevideo. 6, Fasículo 1-2, pp.153-168. 